Stewart, M. J. (1918). Chemical Warfare Medical Committee Report No. 12. London, H.M.S.O

Tallal, L., et al. (1970). Cancer (Philadelphia), 25, 306.

Tan, C., Tasaka, H., and Di Marco, A. (1965). Proceedings of the American

Association for Cancer Research, 6, 64 .
Vaitkevicius, V. C., Brennan, M. J., Beckett, V. L., Kelley, J. E., and Talley, R. W. (1961). Cancer (Philadelphia), 14, 131 .

Vercillo, L., and Esposito, S. (1958). Haematologica, 43, 345.

Waldenström, J. (1964). British Medical fournal, 1, 859.
Weiss, A. J., Jackson, L. G., and Carabasi, R. (1961). Annals of Internal Medicine, 55, 731 .

Welch, A. D. (1961). Cancer Research, 21, 1475.

Whitelaw, D. M., Cowan, D. H., Cassidy, F. R., and Patterson, T. A. (1963). Cancer Chemotherapy Reports, 30, 13.

Wilkinson, J. F., and Fletcher, F. (1947). Lancet, 2, 540

Wiltshaw, E. (1965). Fournal of Obstetrics and Gynaecology of the British Commonwealth, $72,586$.

Wright, T. L., et al. (1963). Cancer Chemotherapy Reports, 23, 169.

\title{
Electroencephalographic Prediction of Fatal Anoxic Brain Damage After Resuscitation from Cardiac Arrest
}

\author{
C. D. BINNIE,* M.D. ; P. F. PRIOR, † M.B., B.S. ; D. S. L. LLOYD, $\ddagger$ B.SC. \\ D. F. SCOTT, $\$ M.B., M.R.C.P., D.P.M. ; J. H. MARGERISON, $\|^{* *}$ M.B., B.CHIR.
}

British Medical fournal, 1970, 4, 265-268

\begin{abstract}
Ummary: Ninety-three electroencephalograms (E.E.G.s) $\checkmark$ were recorded within a week of cardiac resuscitation from 41 patients in whom the subsequent outcome was known to be either recovery of cerebral function or death with associated pathological evidence of gross anoxic brain damage. A statistical analysis of observations on these E.E.G.s yielded a discriminant function for predicting death or survival. Predictions based on each of the 93 individual E.E.G.s would have been correct in 92 and at a confidence level better than $99 \%$. The same discriminant function was found to be applicable to a further 19 patients who died but did not undergo neuropathological studies and to 33 others in whom the clinical picture was complicated by such factors as uraemia or head injury. Thus it seems that the presence or absence of fatal brain damage after cardiac arrest can be reliably predicted from E.E.G.s taken within a week of resuscitation. An estimate of the probability of survival is now routinely included in the clinical report on each E.E.G. taken after cardiac arrest.
\end{abstract}

\section{Introduction}

Studies of the electroencephalogram (E.E.G.) after cardiorespiratory arrest and subsequent resuscitation are chiefly concerned with the isoelectric or "flat" tracing and its ethical and medicolegal implications for the pronouncement of death (Jouvet, 1959; Arfel and Fischgold, 1961; Bickford et al., 1965; Rossoff and Schwab, 1968). Some authors, however, describe E.E.G. signs which may distinguish various types of outcome, with the aim of identifying patients who are capable of useful survival (Hockaday et al., 1965; Pampiglione and Harden, 1968; Prior and Volavka, 1968). While the definition of death remains a fertile topic of ethical debate, the prediction of outcome after cardio-respiratory arrest and resuscitation appears a more profitable subject for scientific inquiry.

After Pampiglione's (1962) description of E.E.G. abnormalities which may follow cardiac or respiratory arrest, Hockaday et al. (1965) showed that death or survival could be predicted by means of the E.E.G. with a reliability of the order of $80 \%$.

\footnotetext{
* Senior Registrar in Clinical Neurophysiology, St. Bartholomew's Hospital, London E.C.1.

+ Medical Assistant, Department of Electroencephalography, the London Hospital, London E.1. Computer Manager, Department of Clinical Neurophysiology, St. Barth-
olomew's Hospital, London E.C.1.

$\checkmark$ Consultant in Charge, Department of Electroencephalography, the London Hospital, London E.1.

\| Consultant Neurophysiologist, St. Bartholomew's Hospital, London
E.C.1. **Dr. Margerison died on 4 August.
}

Pampiglione and Harden (1968) achieved a similar success rate in children. We have for some years tried to apply the criteria described by Hockaday et al. (1965) and, more recently, used those of Prior and Volavka (1968) and of Pampiglione and Harden (1968). Many E.E.G.s seem to be unclassifiable, and in any event the accuracy of prediction does not meet the requirements of the clinical situation. All the above methods involve grading records along a simple scale of increasing abnormality, on the basis of only a few featureschiefly frequency and amplitude-thereby discounting much of the information ordinarily used in assessing an E.E.G.

\section{Methods}

A method of visual assessment and coding was developed to provide a detailed description of any E.E.G. which might be obtained after resuscitation. Fifty-eight tracings from a pilot study at St. Bartholomew's Hospital were examined by one of us (C.D.B.) without reference to clinical information, and scored on 49 variables which described the presence, prominence, and distribution of a wide range of E.E.G. phenomena. Two other variables were admitted-the state of awareness during the recordings, as indicated by neurological evidence, and the time elapsed between resuscitation and the E.E.G. A preliminary statistical assessment suggested that this range of information might provide a satisfactory basis for distinguishing patients who died from those who survived. Training sessions were then carried out to establish an acceptable level of reliability between two observers (C.D.B. and P.F.P.). These observers then applied the same system of rating to all E.E.G.s taken after resuscitation at the London Hospital during the years 1964-8. The result of the analysis of these records is presented here.

\section{Patients}

During the five-year period 127 patients were referred to the London Hospital E.E.G. department because they had failed to regain consciousness rapidly after resuscitation from circulatory or respiratory arrest. In 93 of these subjects legible tracings were obtained within seven days of resuscitation and there had been adequate documentation of the arrest and of the subsequent clinical course. Ninety were adults aged 18 to 89 and the remaining three were less than 10 years old. The 93 patients were divided in three categories (Table I).

Group A.-These 41 adult patients met the following criteria: (1) cardiac arrest was known to have occurred; (2) there was no associated disorder considered likely to complicate the E.E.G. 
assessment-for example uraemia, head injury, drug intoxication; and (3) the outcome was reliably established, either as recovery of consciousness without apparent intellectual or neurological deficit $o r$ as death from anoxic brain damage which was demonstrated at necropsy. Except in two cases with gross macroscopic changes pathological examination included detailed histological studies. In these subjects 44 episodes of cardiac arrest were studied: 23 were followed by recovery and 21 by death with anoxic brain damage. Ninety-three E.E.G.s were taken and the analysis of the findings forms the main body of the present study.

Group B.-These 19 patients had suffered cardiac arrest without complicating factors likely to affect the E.E.G., and died in coma. Though it was thought on clinical grounds that anoxic briin damage had occurred, decisive proof was lacking as neuropathological studies had not been performed.

Group C.-These 33 patients were excluded from groups A and $B$ for one or more of the following reasons: (1) there were associated disorders considered likely to affect the E.E.G.-for example, head injury, uraemia, or drug overdose; (2) the age was less than 18 years; or (3) cardiac arrest was not proved though respiratory arrest or profound hypotension was known to have occurred.

TABLE I.-Clinical Classification and Outcome in 93 Patients After Cardiorespiratory Resuscitation

\begin{tabular}{|c|c|c|c|c|c|c|}
\hline \multirow{3}{*}{$\begin{array}{l}\text { Clinical } \\
\text { Group }\end{array}$} & \multicolumn{3}{|c|}{ Outcome of Each Episode } & \multirow{3}{*}{$\begin{array}{c}\text { Total } \\
\text { Episodes }\end{array}$} & \multirow{3}{*}{$\begin{array}{c}\text { Total } \\
\text { Patients }\end{array}$} & \multirow{3}{*}{$\begin{array}{l}\text { Total } \\
\text { E.E.G.s }\end{array}$} \\
\hline & \multirow[b]{2}{*}{ Recovery } & \multicolumn{2}{|c|}{ Brain Damage } & & & \\
\hline & & $\begin{array}{l}\text { Anoxic Brain } \\
\text { Damage at } \\
\text { Necropsy }\end{array}$ & $\begin{array}{l}\text { Clinical } \\
\text { Evidence } \\
\text { Only }\end{array}$ & & & \\
\hline $\begin{array}{l}\text { A } \\
\text { B } \\
\text { - }\end{array}$ & 23 & 21 & $\begin{array}{l}19 \\
15\end{array}$ & $\begin{array}{l}44 \\
19 \\
33\end{array}$ & $\begin{array}{l}41 \\
19 \\
33\end{array}$ & $\begin{array}{l}93 \\
42 \\
62\end{array}$ \\
\hline & & & & & & \\
\hline
\end{tabular}

\section{E.E.G. Studies}

The E.E.G.s were recorded on either a Kaiser Tr. 608 channel or an Elema Schönander 16-channel electroencephalograph using platinum needles or chlorided silver disc electrodes applied according to the international $10 / 20$ system. If, as was often the case, the clinical situation was considered not to permit application of a full set, a reduced array of only 10 widely-spaced electrodes was used. The results presented are based on E.E.G.s taken from half an hour to seven days after collapse; in practice at least one tracing had usually been obtained within the first 48 hours.

\section{Statistical Analysis}

The visual appraisal of the E.E.G.s from all three groups of patients yielded 197 sets of observations on 51 variables. A correlation matrix showed that these data contained much redundancy; this was reduced by combining linearly correlated variables, so that the original 51 observations on each E.E.G. were replaced by only 36 . A discriminatory analysis was made of the transformed data from the 93 E.E.G.s of group A. This yielded a discriminant function for distinguishing between the records of patients who recovered and the tracings of those with proved anoxic brain damage. Discriminant scores were then computed for the E.E.G.s from all three groups.

\section{Group A}

\section{Results}

The discriminatory analysis of the observations on the 93 E.E.G.s from group $A$ (the 41 patients with uncomplicated cardiac arrest and known outcome) showed pronounced differences between the records of patients who recovered and those of subjects with proved anoxic brain damage. The distributions of the discriminant scores in these two categories are shown in the Chart.

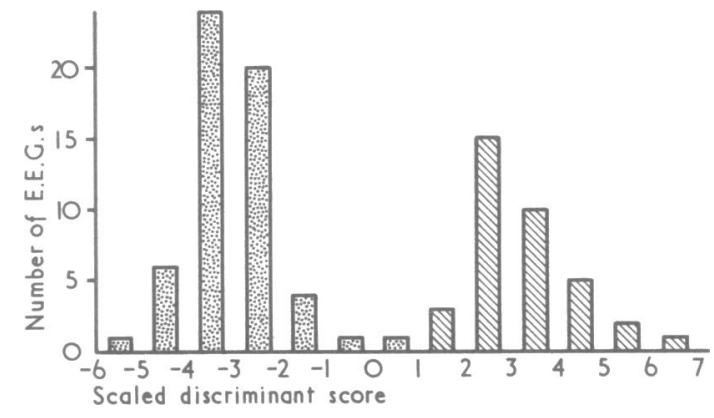

Discriminant scores on 93 E.E.G.s from patients with established outcome. The scores are in arbitrary units scaled so that zero corresponds to equal probabilities of either death or survival. The values on the right of the probabilities of either death or survival. The values on the right of the
figure relate to observations on those who died with anoxic brain damage and those on the left to patients who recovered. The two groups are clearly distinct and only one E.E.G. has been misclassified.

Out of 93 E.E.G.s, only one was misclassified. In this single instance the discriminant score was very close to the value at which death and survival were equiprobable and would not in practice have offered a basis for any clinical decision. Thus if accuracy is considered the chief criterion by which the results should be assessed it seems that, even from a single E.E.G., the outcome in terms of cerebral death or survival could have been predicted with high reliability. In the real clinical situation, however, it is the confidence which can be attached to statements concerning the individual patient which is most important. In each of the 92 correctly classified E.E.G.s the discriminant score would have indicated a probability of survival either less than 0.01 or greater than 0.99 , and in 90 the confidence level would have been better than $99.8 \%$.

E.E.G. prediction is clearly of most practical value while the outcome is still in real doubt-that is, before the patient has either regained consciousness or remained for many hours in profound coma. Of the 93 E.E.G.s on which this analysis was based, 26 were taken from patients who were in stupor or awake and 18 from patients who were in deep coma. Thus the remaining 49 E.E.G.s were recorded at a stage of moderate coma, when physical examination would not have provided a basis for reliable predictions.

\section{Other Groups}

The discriminant function originally developed from group A was also used to calculate scores for group B (the 19 patients with fatal outcome consistent clinically with anoxic brain damage, but without neuropathological confirmation). In most the discriminant scores would have led to the prediction of a fatal outcome (Table II), but out of 42 E.E.G.s, 10 (from seven patients) were classified as suggesting survival. Seven of these 10 records originated from four subiects in whom the immediate cause of death may not have been cerebral, in that they had remained in light coma or stupor for periods ranging

TABLE II.-Predictions Based on 42 E.E.G.s from Group B, 19 Patients who Died and Exhibited Clinical Signs of Brain Damage

\begin{tabular}{c|c|c}
\hline Death & Prediction \\
\hline 25 & $\begin{array}{c}\text { Uncertain } \\
0.01<\mathrm{P}<0.99\end{array}$ & Survival \\
\hline 7 & 10
\end{tabular}

TABLE III.-Predictions Based on 62 E.E.G.s from Group C (33 Patients) Compared with Eventual Outcome

\begin{tabular}{|c|c|c|c|c|}
\hline & \multirow{2}{*}{ Outcome } & \multicolumn{3}{|c|}{ Prediction } \\
\hline & & Death & $\begin{array}{c}\text { Uncertain } \\
0.01<P<0.99\end{array}$ & Survival \\
\hline $\begin{array}{l}\text { Death } \\
\text { Survival }\end{array}$ & $\begin{array}{lll}. & \cdots & \cdots \\
\cdots & \cdots & \end{array}$ & $\begin{array}{r}31 \\
2\end{array}$ & $\begin{array}{l}2 \\
2\end{array}$ & 23 \\
\hline
\end{tabular}


from a week to many months, apparently with brain damage of a degree not incompatible with survival.

The predictions based on discriminant si ores for 62 E.E.G.s from group $C$ (the 33 patients with respiratory arrest, profound hypotension, or various complications) are given in Table III. Thirty-three tracings would have led to a prediction of death, and this would have been correct in 31 instances. The two misclassified records were from patients with barbiturate poisoning, a condition known to confuse the interpretation of the E.E.G. after cardiac arrest (Haider et al., 1968). Survival would have been predicted from 25 records, two of which originated from patients who died. In one of these the clinical circumstances were again helpful, as the tracing was the first of two obtained from a man who remained in coma for some weeks and died eventually in hepatic and renal failure. Cerebral anoxic changes found at necropsy were not extensive. Two other patients in whom survival was correctly predicted had substantial brain damage; one is still in an institution 18 months after the catastrophe, while the other survived for some weeks in a confused state and then suffered a further cardiac arrest which was rapidly fatal, necropsy showing moderate anoxic cerebral pathology. Thus in these three patients from group C and in four others of group B there was presumably nonlethal brain damage and the E.E.G.s were classified with the records of those who recovered. This finding is of interest as it suggests that a similar method of assessment and analysis could possibly be used to identify patients having brain damage of a degree compatible with life, and perhaps ultimately to predict quality of survival.

\section{E.E.G. Signs of Prognostic Value}

Though the E.E.G. phenomena studied had been chosen without known reference to their likely predictive value, the weightings produced by the discriminant analysis gave some indication of their relative importance.

The unfavourable implications of an isoelectric tracing are well known (Silverman et al., 1969) and will not be discussed. When activity was recordable the most adverse sign was the regular recurrence of any particular E.E.G. phenomenon. The slower the repetition rate the worse was the prognosis. Paroxysmal activity (spikes, either single or multiple, sharp waves, or complexes of these together with slower components), though rarely encountered, was an unfavourable sign. An E.E.G. of consistently low amplitude-for example, 10-20 $\mu \mathrm{V}-$ was also an adverse finding, even though the tracing was not isoelectric. Similarly, episodic reduction in amplitude, however slight in degree, signified a poor prognosis. Transients of any nature were also ominous; they were typically of simple stereotyped wave form, either monophasic or biphasic, and of too great duration to be classified as sharp waves. Other features which were less consistently associated with fatality were asymmetries, a lack of theta and of alpha activity, and the absence of an E.E.G. response to painful or auditory
stimulation.

The dominant frequency which plays such a conspicuous part in Hockaday's and other classifications made surprisingly little contribution to the discriminant score. Of particular interest in this context is a specific clinical and E.E.G. pattern which was often seen. A patient in deep coma might have an E.E.G. consisting mainly of rhythmic activity within the alpha range, of low amplitude, and unresponsive to stimuli. If classifiable at all by existing criteria such a record would have been graded as indicating a good prognosis. In fact the outcome was invariably fatal and was correctly so predicted from the discriminant score. Similar findings have been described in patients with vascular disorders of the brain stem (Loeb and Poggio, 1953; Lundervold et al., 1956; Chase et al., 1968) and with head injuries (Whelan et al., 1955; Chatrian et al., 1964; Bickford and Klass, 1966) but not apparently after cardiac
arrest.

\section{Discussion}

When the outcome of known cardiac arrest was established as well as might be (group A) death or survival could apparently have been reliably predicted on the basis of a single E.E.G., and usually with a confidence level better than $99.8 \%$. Nevertheless, it should be emphasized that the techniques used were far from optimal. During the early stages of the period of data collection the E.E.G.s were taken by technicians who had no special experience of recording from unconscious patients in an intensive care unit, and consequently the tracings were often of poorer quality and of shorter duration than those more recently obtained. Furthermore, serial changes in the E.E.G. were not considered though they are generally held to be of prognostic value (Pampiglione and Harden, 1968). The method of visual assessment was intended to provide a fairly comprehensive description of each E.E.G., but there is no reason to suppose that either the choice of variables or the system of scoring was ideal. Indeed the most encouraging feature of this study may be that successful prediction has proved possible despite obvious limitations. That the E.E.G. is a powerful tool for predicting the outcome after cardio-respiratory resuscitation must now be regarded as incontrovertible.

The coding of the primary E.E.G. observations is complex, but probably requires less skill and judgement than do previous methods of categorization. The ability to assess E.E.G.s obtained after cardiac arrest need not, therefore, be vested in one specially experienced member of a department. Indeed, since completion of this study a familiarity with the E.E.G. signs of most prognostic value and a detailed knowledge of the weightings assigned to them has not enabled us to make subjective predictions with a reliability approaching that achieved by use of the discriminant score.

The results in group $C$ suggest that useful prediction can generally be made even in the presence of various complicating factors known to affect the E.E.G. This finding, however, in no way absolves the electroencephalographer from the need to consider the clinical circumstances, as, for example, hypothermia or barbiturate intoxication, in formulating his final opinion. It would, moreover, be rash to generalize beyond the range of the present clinical material, and caution will be necessary whenever new methods of treatment or additional complications are encountered.

We hope those who accept these findings and conclusions will test, utilize, and improve our methods and so refine the predictions as to provide information about the quality as well as the fact of survival. In our view, however, no useful purpose would be served by publication of detailed criteria for assessing the records or of the weightings required to calculate the discriminant score, since E.E.G. terminology is at present far from precise and no simple verbal account could be expected to enable another observer to rate E.E.G.s in precisely the same manner. Any systematic difference in the analysis of the primary data would obviously invalidate a discriminant score obtained from the weightings calculated in the present investigation. Those who wish to use these techniques for clinical purposes must necessarily carry out a similar analysis of their own material. A routine service for predicting outcome following resuscitation has now been established at St. Bartholomew's Hospital. When an E.E.G. is assessed for clinical purposes the discriminant score is calculated and a statement of the probability of survival is included in the report.

The results of this study are considered to support a general contention of ours (Binnie et al., 1966) that adequate use is rarely made of the human capacity for recognizing E.E.G. patterns.

We are grateful to Professor H. Urich, of the London Hospital, for carrying out the neuropathological studies; to Professor V. I. Price, of the City University, for help and for the use of his department's I.C.L. 1905 computer; and to Professor J. F. Scott, of Sussex University, for statistical advice. 
Arfel, G., and Fischgold, H. (1961). Electroencephalography and Clinical Neurophysiology, 13, 653

Bickford, R. G., Dawson, B., and Takeshita, H. (1965). Electroencephalography and Clinical Neurophysiology, 18, 513.

Bickford, R. G., and Klass, D. W. (1966). In Head Injuries ed. W. F. Caveness and A. E. Walker, p. 63. Philadelphia, Lippincott.

Binnie, C. D., Margerison, J. H., and Whitehead, M. K. (1966). Electroencephalography and Clinical Neurophysiology, 21, 309.

Chase, T. N., Moretti, L., and Prensky, A. L. (1968). Neurology (Minneapolis), 18, 357.

Chatrian, G. E., White, L. E., and Shaw, C. M. (1964). Electroencephalography and Clinical Neurophysiology, 16, 285 .

Haider, I., Oswald, I., and Matthew, H. (1968). British Medical Fournal, 3,314 .

Hockaday, J. M., Potts, F., Epstein, E., Bonazzi, A., and Schwab, R. S. (1965). Electroencephalography and Clinical Neurophysiology', 18, 575.
Jouvet, M. (1959). Electroencephalography and Clinical Neurophysiolugy, $11,805$.

Loeb, C., and Poggio, G. (1953). Electroencephalography and Clinical Neurophysiology, 5, 295.

Lundervold, A., Hauge, T., and Löken, A. C. (1956). Electroencephalography and Clinical Neurophysiology, 8, 665.

Pampiglione, G. (1962). Proceedings of the Royal Society of Medicine, 55, 653. Pampiglione, G., and Harden, A. (1968). Lancet, 1, 1261.

Prior, P. F., and Volavka, J. (1968). Electroencephalography and Clinical Neurophysiology, 24, 593 .

Rossoff, S. D., and Schwab, R. S. (1968). Electroencephalography and Clinical Neurophysiology, 24, 283.

Silverman, D., Saunders, M. G., Schwab, R. S., and Masland, R. L. (1969).

Fournal of the American Medical Association, 209, 1505.
Whelan, J. L., Webster, J. E., and Gurdjian, E. S. (1955). Elcctroencephalography and Clinical Neurophysiology, 7, 495.

\title{
Immunity, Transferrin, and Survival in Kwashiorkor
}

\author{
H. McFARLANE, ${ }^{*}$ PH.D., M.R.C.PATH. ; S. REDDY, $†$ M.B., B.S., M.R.C.P.ED. ; K. J. ADCOCK, $\ddagger$ M.B., B.S., D.R.C.o.G. \\ H. ADESHINA, $\$ A.M.I.L.T. ; A. R. COOKE, $\ddagger$ M.B., B.S., D.T.M. \& H. ; J. AKENE
}

\begin{abstract}
Gummary: In a study of 40 children with kwash$\checkmark$ iorkor, serum albumin, transferrin, and immunoglobulin levels were measured. Treatment included chloroquine, pyrimethamine, multivitamins, folic acid, iron compounds, and a high-protein diet. After two weeks the mean serum transferrin values in the children who survived and those who died were $1.30 \mathrm{mg} . / \mathrm{ml}$. and $0.33 \mathrm{mg} . / \mathrm{ml}$., respectively. Many of the children died immediately after treatment started, and it is suggested that in children with severe kwashiorkor and low serum transferrin levels any increase in free-circulating iron may result in overwhelming infection and death. Thus the appropriate time for instituting iron therapy in such patients should be reconsidered.
\end{abstract}

\section{Introduction}

In kwashiorkor assessment of the degree of malnutrition or its prognosis on clinical grounds alone is universally accepted as being difficult. Hence several attempts have been made to find suitable biochemical tests to fulfil this need (Waterlow et al., 1960). The low serum transferrin levels found in kwashiorkor may be significant (Antia et al., 1968) and of several biochemical tests McFarlane et al. (1969) found the serum transferrin to be the most accurate index for assessing kwashiorkor.

Infection is more prevalent in this disease than in the rest of the population (Scrimshaw et al., 1960), and there is evidence of an immune deficiency state in kwashiorkor. Brown and Katz (1965), in Uganda, noted that the serum immunoglobulin concentration in kwashiorkor was severely depressed, whereas Najiar et al. (1969) observed high serum IgG, IgA, and IgM in marasmic infants with no clinical evidence of kwashiorkor. On the other hand, mean levels of serum immunoglobulin values in both severe and moderate kwashiorkor did not show much difference (McFarlane et al., 1970), though some individual kwashiorkor children had reduced serum immunoglobulin values. We report here the results of the Heaf test and a sequential study of

\footnotetext{
* Late Professor of Chemical Pathology, University of Ibadan. Present address: Department of Chemical Pathology, Manchester University, Manchester, England.

† Head of General Outpatients.

Senior Hospital Medical Officer, General Outpatient Department.

$\$$ Senior Technician.

II Laboratory Assistant (Research).

University College Hospital, Ibadan, Nigeria.
}

albumin, transferrin, and immunoglobulins in children with kwashiorkor.

\section{Patients and Methods}

Of the 40 children studied with kwashiorkor, who were attending the general outpatient clinic of University College Hospital, Ibadan, 21 were girls and 19 boys aged $1 \frac{1}{2}$ to 5 years. Of these seven had hepatomegaly, one of whom also had splenomegaly; only one child was jaundiced; and almost all had varying degrees of diarrhoea, two having malaria. Ascaris ova were found in the stools of two children. Clinical evidence of bacterial infection was found in four childrentwo had otitis media, one had conjunctivitis and one had furunculosis.

The Heaf test was done with the standard technique and was negative in all cases. No abnormality was detected on routine chest $x$-ray examination. Twenty-nine were haemaglobin genotype AA. All the children were treated initially with chloroquine and thereafter with weekly doses of pyrimethamine; a multivitamin mixture plus $5 \mathrm{mg}$. of folic acid was given daily. The children were given a high-protein diet in the form of milk powder that was known to have a high-protein content. Sulphadimidine and potassium chloride were prescribed for those with diarrhoea, and the bacterial infections noted above were treated with antibiotics as indicated. Oral ferrous sulphate was prescribed for most of those who had low packed cell volume after the diarrhoea had subsided. When oral iron was not available intramuscular iron was given. Most of the children were followed up for six weeks or more, though many died or dropped out of the study. All were seen every other day during the first two weeks and thereafter once or twice a week, depending on the severity of the illness.

Serum proteins were estimated by the method of Gornall $e t$ al. (1949), serum uric acid by the method of Feichtmeir and Wrenn (1955), and serum transferrin and immunoglobulins by the Hyland immunoplate method.

\section{Results}

\section{Serum Albumin and Serum Transferrin}

The time course sequence of albumin and transferrin concentration in the children with kwashiorkor who survived and 